\title{
Looking at Cell Mechanics with Atomic Force Microscopy: Experiment and Theory
}

\author{
RAFAEL BENITEZ, ${ }^{1}$ AND JOSÉ. L. TOCA-HERRERA ${ }^{2 *}$ \\ ${ }^{1}$ Department of Mathematics, University Center of Plasencia, University of Extremadura, Avda. Virgen del Puerto 2, 10600 Plasencia, \\ Spain \\ ${ }^{2}$ Institute for Biophysics, Department of Nanobiotechnology, University of Natural Resources and Life Sciences Vienna (BOKU), Muth- \\ gasse 11, 1190 Vienna, Austria
}

KEY WORDS atomic force microscopy; cell mechanics; Young modulus; relaxation time; contact mechanics; viscoelasticity; Hertz and JKR models

\begin{abstract}
This review reports on the use of the atomic force microscopy in the investigation of the mechanical properties of cells. It is shown that the technique is able to deliver information about the cell surface properties (e.g., topography), the Young modulus, the viscosity, and the cell the relaxation times. Another aspect that this short review points out is the utilization of the atomic force microscope to investigate basic questions related to materials physics, biology, and medicine. The review is written in a chronological way to offer an overview of phenomenological facts and quantitative results to the reader. The final section discusses in detail the advantages and disadvantages of the Hertz and JKR models. A new implementation of the JKR model derived by Dufresne is presented. Microsc. Res. Tech. 77:947-958, 2014. @ 2014 Wiley Periodicals, Inc.
\end{abstract}

\section{INTRODUCTION}

According to the Web of Science the oldest article concerning cell mechanics is (Table 1; Fischel, 1906). The same database tells us that up to date there are more that 18,000 articles about cell mechanics; although barely 300 refer to cell mechanics studied with atomic force microscopy (AFM). For the same search criteria, Scopus database yields a result of about four hundred manuscripts. These numbers could indicate that the field still has a long way to go, although the first articles date from the early 1990s. At this point, it is worth mentioning the work developed by: (i) Henderson et al., who measured the dynamics of actin filaments on glial cells (Henderson et al., 1992), (ii) Putman et al., who quantified the time-dependent relaxation of a monkey kidney cell surface in liquid with cantilevers of spring constant of about 0.58 N/m (Putman et al., 1994), (iii) Braunstein et al., who tracked cytoskeletal elements and organelles of quiescent and activated Rat Basophilic Leukemia cells (Braunstein and Spudicht, 1994), (iv) Hoh and Schoenenberger, who reported on the surface morphology and mechanical properties of Madin-Darby canine kidney (Hoh and Schoenenberger, 1994), and (v) Radmacher et al., who quantified the Young modulus of human platelets with a resolution of $100 \mathrm{~nm}$ (Radmacher et al., 1996).

We shall see in the pages to come, what different researches have been discovering about this exciting topic and the way they have done it (in part due to the development of the AFM by commercial companies). An interesting approach to the study of cell mechanics is to consider that the basic structure of the cell can be reduced to fluid sheets enclosing both the cell itself (e.g., phospholipid bilayers) and its compartments, and to filament networks which maintain the shape of the cell (e.g., actin). In contrast to hard materials (e.g., a bridge), cells are soft and the description of their mechanical properties needs theoretical frameworks, which should take into account the molecular nature of the constituents of the cell. This theoretical aspect, in combination with the development of novel experimental devices capable to measure simultaneously different mechanical aspects of cells as a whole, is part of the challenge for the future.

In this short review, we will briefly introduce the atomic force microscope as an imaging and mechanical machine; we will report extensively about the mechanical properties of bacteria and human cells, and finally we will discuss different theoretical methods that are currently used to quantify the mechanical properties of cells.

\section{AFM AS AN IMAGING MACHINE}

In comparison with the transmission and the scanning electron microscopes, the AFM is more versatile when the nanostructure of biomaterials in aqueous environment at different temperatures is investigated (Alessandrini and Facci, 2005; Moreno-Flores and Toca-Herrera, 2013).

Figure 1 shows the main parts of an AFM. The sensing element is a flexible cantilever with a sharp tip at

\footnotetext{
*Correspondence to: José L. Toca-Herrera, Institute for Biophysics, Department of Nanobiotechnology, University of Natural Resources and Life Sciences Vienna (BOKU), Muthgasse 11, A-1190 Vienna, Austria.

E-mail: jose.toca-herrera@boku.ac.at

Received 23 June 2014; accepted in revised form 25 July 2014

REVIEW EDITOR: Prof. Alberto Diaspro

Conflict of Interest: The authors do not have any conflict of interest.

Contract grant sponsor: Etortek Programme, Basque Government; Contract grant number: 07/27-IE07/201; Contract grant sponsor: Spanish Government; Contract grant number: CTQ2007-66541; Contract grant sponsor: IGS program of the Austrian Government.

DOI $10.1002 /$ jemt.22419

Published online 5 August 2014 in Wiley Online Library (wileyonlinelibrary.com).
} 
TABLE 1. Interesting and important articles concerning cell mechanics that are not directly quoted in the text

Kasas S, Gotzos V, Celio MR. 1993. Observation of living cells using the atomic force microscope. Biophys J 64:539-544.

Wu HW, Kuhn T, Moy VT. 1998. Mechanical properties of L929 cells measured by atomic force microscopy: effects of anticytoskeletal drugs and membrane crosslinking. Scanning 5:389-397.

Matzke R, Jacobson K, Radmacher M. 2001. Direct, high-resolution measurement of furrow stiffening during division of adherent cells Nat Cell Biol 3:607-610.

Hengsberger S, Kulik A, Zysset P. 2002. Nanoindentation discriminates the elastic properties of individual human bone lamellae under dry and physiological conditions. Bone 30:178-184.

McElfresh M, Baesu E, Balhorn R, Belak J, Allen M J, Rud RE. 2002. Combining constitutive materials modeling with atomic force microscopy to understand the mechanical properties of living cells, PNAS 99:6493-6497.

Costa KD. 2003. Single-cell elastography: probing for disease with the atomic force microscope. Dis Markers 19:139-154.

Sen S, Subramanian S, Discher DE. 2005. Indentation and adhesive probing of a cell membrane with AFM: theoretical model and experiments. Biophys J 89:3203-3213.

Lulevich V, Zink T, Chen HY, Liu FT, Liu GY. 2006. Cell mechanics using atomic force microscopy-based single-cell compression. Langmuir 22:8151-8155.

Rosenbluth MJ, Lam WA, Fletcher DA, 2006. Force microscopy of nonadherent cells: a comparison of leukemia cell deformability. Biophys. J. 90:2994-3003.

Sokolov I, Iyer S, Woodworth CD. 2006. Recovery of elasticity of aged human epithelial cells in vitro. Nanomedicine 2:31-36.

Kidoaki S, Matsuda T. 2007. Shape-engineered fibroblasts: cell elasticity and actin cytoskeletal features characterized by fluorescence and atomic force microscopy. J Biomed Mater Res A 81:803-810.

Radmacher M. 2007. Studying the mechanics of cellular processes by atomic force microscopy. Methods Cell Biol 83:347-372.

Kuznetsova TG, Starodubtseva MN, Yegorenkov NI, Chizhik SA, Zhdanov RI. 2007. Atomic force microscopy probing of cell elasticity. Micron 38:824-833.

Spagnoli C, Beyder A, Besch SR, Sach F. 2007. Drift-free atomic force microscopy measurements of cell height and mechanical properties. Rev Sci Instrum 78:036111.

Unnikrishnan GU, Unnikrishnan VU, Reddy JN. 2007. Constitutive material modeling of cell: a micromechanics approach. J Biomech Eng 129:315-323.

Pelling AE, Horton MA. 2008. An historical perspective on cell mechanics. Pflugers Arch 456:3-12.

Iyer S, Gaikwad RM, Subba-Rao V, Woodworth C. 2009. Atomic force microscopy detects differences in the surface brush of normal and cancerous cells. Nat Nanotechnol 4:389-393.

Roduit C, Sekatski S, Dietler G, Catsicas S, Lafont F, Kasas S. 2009. Stiffness tomography by atomic force microscopy. Biophys $J$ 97:674-677.
One of the first articles reporting the use AFM on cells. The authors studied the dynamics of the membranes of living cells. They also used scanning electron microscopy and microcinematography as supporting methods.

The authors investigated the architecture of the cytoskeleton and the role of its components by measuring the elasticity, viscoelasticity, and plasticity of L929 cells. The results provided evidence that the cell membrane and the cytoskeleton are mechanically coupled.

Force mapping was used to monitor dynamic changes in the stiffness of the cortex of adherent cells. The authors found that polar relaxation of cells was not necessary for cell division.

$\mathrm{AFM}$ and nanoindentation were used to characterize bone surface and the bone extracellular matrix at dry and physiological conditions. The article also reports on the structural differences between thick and thin lamellae.

This article presented a model to distinguish the mechanical response of the cell from the local surface interactions (e.g., molecular recognition). The proposed model took into account the mechanics of the biomembrane and cytoskeleton.

The authors reported on the combination of imaging and indentation to map the cell mechanical properties. It is also mentioned the need for new analytical methods.

In this study, the reader will find functionalized AFM tips (with SIRPalpha) to probe its native receptor CD47. The work reported not only on specific interactions and unbinding processes but also on membrane indentation. The work showed that the indentation depth takes into account the membrane tension (not following the standard Hertz model).

The article reported on the use of AFM, bright-field and confocal laser scanning microscopy. The authors proposed a simple model consisting of nonpermeable balloon filled with incompressible fluid to obtain the Young's modulus and the bending constant of cell membranes.

In this work, it is shown how to characterize nonadherent cells by immobilizing them in microwells. The authors studied the deformability of human myeloid and lymphoid leukemia cells and neutrophils.

The authors used AFM and immunofluorescence microscopy to study cell mechanics changes during aging in vitro, and its potential relevance for treating the loss of elasticity in epithelial tissues.

AFM and fluorescence microscopy were used to characterize round and spindle-shaped cells cultured on surfaces prepared with photolithography.

The authors found that cell shape affects the elasticity of elongated cells rather than structural characteristics of the stress fibers.

Since cell mechanics is important for numerous cell processes, this article offers a very good overview about the cell components and the use of the AFM to assess the elastic properties of cells. The article also introduces the reader to simple models to evaluate the Young modulus.

This is a nice review that will introduce the reader to the basics of AFM and its application to the investigation of the mechanical properties of cells. The authors made a good selection of references concerning cell aspects such as differentiation, aging, electromotility, or cell pathology.

In this article, the authors discussed the drift problem associated to long duration AFM experiments. The manuscript presents a software that should eliminate the cantilever's drift. The method was tested on rat astrocytes.

The authors proposed a method to correlate the mechanical behavior of the cell with the cell composition. They focused on the fact that the cytoplasm is not homogenous. The model was based on the Mori-Tanaka method of homogenization considering the cell as a fiber-reinforced composite medium satisfying the continuum hypothesis.

An interesting article to read, where the authors commented on early literature presenting an historical framework. The authors also discussed the different modern approaches to get insight about the mechanical properties of cells.

This work reported on the quantitative differences between normal and cancerous human cervical epithelial cells by considering the brush layer on the cell surface. The authors also reported on the differences in brush length between normal and cancerous cells.

The focus of this article was to present a new technique to distinguish structures of different stiffness buried into the bulk of the sample of interest. They authors used finite element models to test the new imaging technique (e.g., rheology at short timescales). 
TABLE 1. Continued

Chaudhuri O, Parekh SH, Lam WA, Fletcher DA. 2009. Combined atomic force microscopy and side-view optical imaging for mechanical studies of cells. Nat Methods 6:383-387.

Donhauser ZJ, Jobs WB, Binka EC, 2010. Mechanics of microtubules: effects of protofilament orientation. Biophys J 99:1668-1675.

Ikai A. 2010. A review on: Atomic Force Microscopy Applied to Nanomechanics of the Cell. Adv. Biochem Eng Biotechnol 119:47-61.

Müller D J, Dufrene IF. 2011. Atomic force microscopy: a nanoscopic window on the cell surface. Trends cell Biol 21:461-469.

Franze K. 2011. Atomic force microscopy and its contribution to understanding the development of the nervous system. Curr Opin Genet Dev 21:530-537.

Lam WA, Chaudhuri O, Crow A, Webster KD, Li T D, Kita A, Huang J, Fletcher DA. 2011. Mechanics and contraction dynamics of single platelets and implications for clot stiffening. Nat Mater 10:6166.

Stroka KM, Aranda-Espinoza H. 2011. Effects of Morphology vs. CellCell Interactions on Endothelial Cell Stiffness. Cell Mol Bioeng $4: 9-27$.

Nawaz S, Sanchez P, Bodensiek K, Li S, Simons M, Schaap AT. 2012. Cell Visco-Elasticity Measured with AFM and Optical Trapping at Sub-Micrometer Deformations. PLoS ONE 7(9): e45297.

Okajima T. 2012. Atomic force microscopy for the examination of single cell rheology. Curr Pharm Biotechnol 13:2623-2631.

Kasas S, Longo G, Dietler G. 2013. Mechanical properties of biological specimens explored by atomic force microscopy. J Phys D 46:133001.

Barreto S, Clausen CH, Perrault C M, Fletcher DA, Lacroix D. 2013. A multistructural single cell model of force-induced interactions of cytoskeletal components. Biomaterials 34:6119-6126.

Picas L, Rico F, Deforet M, Scheuring S. 2013. Structural and mechanical heterogeneity of the erythrocyte membrane reveals hallmarks of membrane stability. ACS Nano 7:1054-1063.

Varghese Chacko J, Canale C, Harke B, Diaspro A. 2013. SubDiffraction Nano Manipulation Using STED AFM. PLoS ONE 8(6):e66608.

Zhou EH, Martinez FD, Fredberg JJ. 2013. Cell rheology: mush rather than machine. Nat Materials 12:184-185.

the end. The deflection of the cantilever, which is due to the interaction forces between the tip and the sample, is measured after detecting the reflected laser beam with a position detector (photodiode). A piezoscanner moves the cantilever along the three dimensions.* The topography of the sample is obtained from the tip/sample interaction and the final image is deliv-
In this work, the authors combined atomic force microscopy with side-view fluorescent imaging to image cellular deformation and cytoskeletal rearrangements. The technique permitted to visualize cell shape under mechanical load, formation of membrane tethers and stress-fiber formation.

This work reported on the application of finite element modeling to microtubule radial deformation. The modeling is correlated with AFM indentation measurements.

This review reported on cell biology aspects such as the mechanical basis of the cellular structure and its interaction with the extracellular matrix.

The review also covered recent advances on nanomechanics.

A review that it is worth reading. The authors showed in which way AFM can be a versatile platform for imaging and manipulating living cells to single-molecule resolution. The discussion is also extended to important issues of cell biology, such as signaling, cell adhesion, or cell division. The authors did not forget to mention the limitation that the technique and the research challenge to come.

This review is a summary about the main work done on the mechanics of nervous cells. The authors pointed out the new directions for the application of the AFM in the field.

The authors studied the mechanics and dynamics of single platelets. They pointed out the relation between clots and strain stiffening of fibrin. This review is an example of how mechanical measurements can be used to investigate physiologic systems.

The articles reported on the measurement of the Young's modulus of live human umbilical vein endothelial cells. The authors observed that cell stiffness increased for larger cell contact areas.

Cell-cell interactions seem to be an important parameter for determining the mechanics of individual cells in tissues.

The authors investigated the response of single 3T3 fibroblasts to different indentations (and load forces). Interestingly they presented an optical trap to indent the cell in the vertical direction. The results showed that at small deformations the elastic modulus is mainly determined by the actin cortex, while at higher indentations viscous effects increased the apparent elastic modulus.

This review summarized the use of AFM to investigate single cell rheology in frequency and time domains. The authors discussed the universality of the cell rheology data extracted with AFM.

In this topical review, the authors outlined the most relevant works related to the application of the AFM as nanomechanical machine to the fields of biology, microbiology, and medicine. The reader will find literature related to viruses, plant cell, or mammalian cells. The article also offers a very good overview of the AFM operating principles and the main models used to quantify the cell mechanical properties.

The authors carried out numerical simulations (with finite elements) on data obtained from AFM experiments. The simulations pointed out that actin cortex and microtubules are the major components targeted in resisting compression.

The authors investigated the relation between imaging and mechanical mapping to understand the cell integrity and function. A major conclusion of the article is that red blood cell membrane mechanics might be regulated by the metabolic state and the assembly of its tructural elements.

The authors combine stimulated emission depletion (STED) microscope with atomic force microscopy. The article provides a good academic microscopy basis for the newcomer and compares the performance of STED with confocal microscopy. From the outcome, it seems that the STED-AFM combination is probably the most accurate, STED works below the diffraction limit, to (nano)manipulate any type of cell, and therefore to carry out accurate cell mechanics experiments.

The article reported on the fact that the cytoplasm of living cells responds to deformation in a similar way as a water-filled sponge. The authors pointed out that this behavior is still an open question in cell mechanics.

ered by a computer. The most popular imaging modes are contact mode and tapping mode. In contact mode, the value of the repulsive force between tip and sample is kept fixed during the scanning of the sample. In tapping mode, the cantilever is oscillating at its resonant frequency (or close to). When approaching the sample, the tip comes into intermittent contact with the 


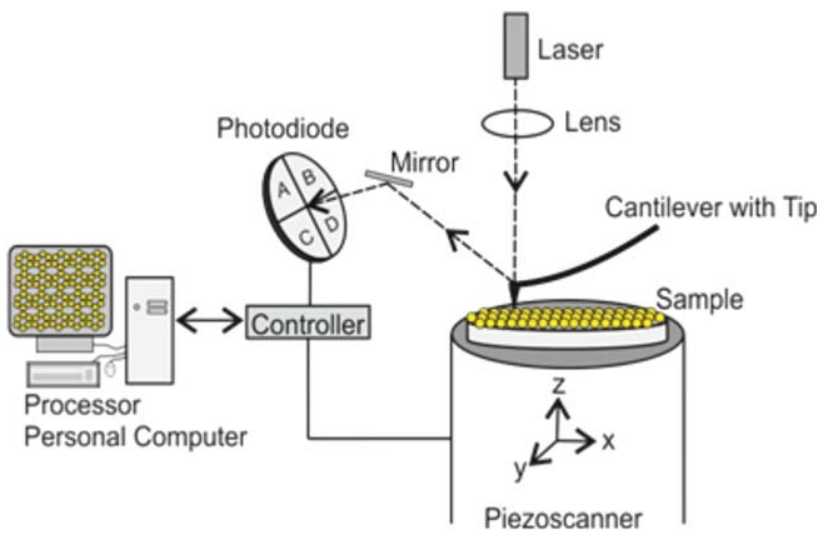

Fig. 1. Schematic drawing of an AFM. [Color figure can be viewed in the online issue, which is available at wileyonlinelibrary.com.]

surface and the tip-sample interactions reduce the amplitude of the oscillations. The amplitude is used as a feedback signal for topographic imaging. In this way, by recording the difference between the phase of the (set) drive signal and the phase of the cantilever response, a "phase" image is obtained. This type of image delivers information about the viscoelastic and adhesive properties of the sample (Garcia et al., 1999; Radmacher et al., 1992). This imaging mode has been able to deliver, for example, refined structural information of cells (Camesano et al., 2000) and polymers (Hobbs et al., 2009). Tapping mode, in comparison with contact mode, presents the advantage of reducing friction forces when scanning (soft) samples.

Cell imaging (and cell mechanics) experiments require especial care, for example, about cell immobilization and viability and the strength of the scanning force. Cell immobilization can be achieved, for example, by unspecific interactions (surface charge) or by enzyme-directed protein immobilization (Murphy et al., 2004; Saravia and Toca-Herrera, 2009).

Today, commercial AFM fluid cells allow us to measure at $37^{\circ} \mathrm{C}$ in different buffer solutions solving possible cell viability problems. Finally, the scanning force should be as low as possible to avoid sample damaging, that implies the use of soft cantilevers of spring constant values between 0.01 and $0.06 \mathrm{~N} / \mathrm{m}$ (Doktycz et al., 2003; Le Grimellec et al., 1998).

\section{AFM AS A MECHANICAL MACHINE}

The atomic force microscope can also be used as a "mechanical" machine allowing the investigation of adhesion and surface forces (Borkovec et al., 2012; Puech et al., 2006), polymer elasticity (Bornschlögl and Rief, 2011; Fisher et al., 1999), ligand-receptor forces (Florin et al., 1994; Hugel and Seitz, 2002; Hinterdorfer and Dufrêne, 2006), or cell mechanics (Radmacher, 2002; Alcaraz et al., 2003; Scheuring and Dufrene, 2010; Melzak et al., 2012; Vargas-Pinto et al., 2013).

In force-distance experiments, an AFM-tip or a colloidal probe (Ducker et al., 1991) is extended toward and retracted from the sample at speeds that may vary between $50 \mathrm{~nm} / \mathrm{s}$ and $10 \mu \mathrm{m} / \mathrm{s}$ (strictly speaking, the speed of the tip is governed by the movement of the piezo). During this process, the deflection of the cantilever is determined as a function of the displacement of the piezo-scanner (Moreno-Flores and Toca-Herrera, 2013) and the force sensed by the cantilever is calculated using Hooke's law (which is equal to the cantilever deflection times its spring constant). The spring constant of the cantilever should be calculated in every experiment. A review of the different experimental calibrating methods can be found in (Cumpsen et al., 2008). The obtained force-curve curves can be divided in three parts: approach, contact with the sample, and retraction. The approaching curve delivers information about repulsive or attractive forces between tip/colloidal probe and sample (e.g., electrostatic, van der Waals, hydration, or entropic forces). In the second part of the curve, the cantilever is in contact with the sample (e.g., cell) providing information about its mechanical properties (e.g., Young's modulus, relaxation time, and viscosity). Finally, the retracting curve gives information about adhesion forces, the existence of tethers and possible molecular unfolding events. In this review, we will focus on the second part of the force curve (see Fig. 2).

The AFM cannot measure absolute distances between the tip and the sample of interest. The separation between both is derived from the position given by the piezoelectric scanner. To quantify the type of interaction or physical quantity related to the mechanical properties it is necessary to detect the contact point between tip and sample.

Some approaches have been proposed. Van Landingham et al. (2001) reported a summary of classical approaches applied to polymers and its influence on their mechanical characterization. Crick and Yin (2007) took into account the noise contribution to implement the information about linear elastic materials of different Young moduli (Crick and Yin, 2007). Benitez et al. proposed an algorithm, based on the statistical analysis of the slope changes of the curve (Benitez et al., 2013), and compared it with two other different methods, based on a pure elastic best fit and on an extrapolation to zero load of the sample deformation (Melzak et al., 2010). Force-distance curves are the most common, and probably, intuitive way to assess the mechanical properties of cells (e.g., Young modulus). However, we will also see that, following classical rheology, force relaxation, and creep experiments are starting to be popular within the AFM community since they also deliver information about relaxation times and viscosities of the different cell parts (Vadillo-Rodriguez et al., 2008; Moreno-Flores et al., 2010a,b; Lu et al., 2014).

\section{MECHANICAL PROPERTIES OF CELLS MEASURED WITH AFM}

As stated in the introduction, the first publications about the mechanical properties of cells with AFM date from the early $1990 \mathrm{~s}$. In this part, we will quote some representative work carried out on prokaryotic and eukaryotic cells, being the latter the most extensively studied so far.

\section{Prokaryotic Cells}

In 2000, Arnoldi et al. measured the deformability of a bacterial wall of the species Magnetospirillum gryphiswaldense using force-distance curves; the authors 


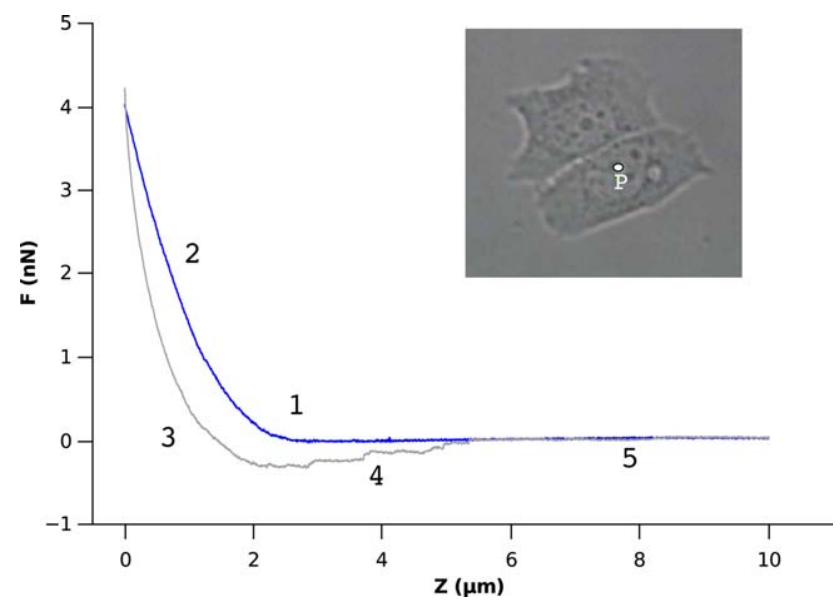

Fig. 2. Representative force-distance curve performed on a breast cancer cell (MCF-7) at point $(\mathrm{P})$. The blue line denotes the approaching curve: the curvature (1) indicates the existence of a repulsive force between tip and sample; in (2) the tip is in contact with the cell, and information about its mechanical properties can be obtained. The gray line is the retracting curve: (3) shows adhesion forces, hysteresis behavior, and tether (4). At (5) the cantilever is at rest (the tip does not interact with the sample). [Color figure can be viewed in the online issue, which is available at wileyonlinelibrary.com.]

also derived a theoretical expression for the force exerted by the wall on the cantilever as a function of the indentation. They determined that the turgor pressure oscillated in a range from 85 to $150 \mathrm{kPa}$ (Arnoldi et al., 2000). Another interesting and complementary work was related to the study of the nanomechanical properties of Shewanella putrefaciens at two different $\mathrm{pH}$ values. The authors found that the approaching force-distance curves showed nonlinear and linear regimes that were related to the progressive indentation in the bacterial cell wall, concluding that the variations in nanomechanical properties (Young modulus) was due to the response of the bacterial surface to the $\mathrm{pH}$ variation (Gaboriaud et al., 2005). In 2006, Mendez-Vilas et al. found that Staphylococcus epidermidis presented two different mechanical regimes. The first regime was located in the outer part of the cell wall (about $48 \mathrm{~nm}$ ) and its relative elasticity was about a third of the relative elasticity of the inner part of the cell wall. In addition, force-distance curves showed hysteresis in the inner part, indicating viscoelasticity behavior (Mendez-Vilas et al., 2006). Further studies have examined the cell envelope of (gram-negative bacteria) Pseudomonas aeruginosa PAO1 from another perspective: performing creep experiments. A constant compressive force is applied on the bacteria to record the time-dependent indentation due to its viscoelastic properties (an experimental limitation for such experiments might be due to the nonlinearity behavior of the used piezo scanner). Interestingly, the authors proposed a mechanical model to describe the effective cell spring constant and the characteristic time of the creep deformation (see Fig. 3). Furthermore, the researchers showed that the effective spring constant increased after cross-linking with glutaraldehyde (Vadillo-Rodriguez et al., 2008).

In 2010, Raman et al. proposed the use of a dynamic method to map the local stiffness, the stiffness gradient, and the viscoelastic dissipation of live Escherichia coli bacteria, rat fibroblasts and human red blood cells in buffer solutions. These physical properties were obtained from the zeroth, first, and second harmonic components of the Fourier spectrum of the AFM cantilevers interacting with the cell surface (Raman et al., 2010).

An interesting experiment combining microfluidics and AFM to investigate biofilm behavior was performed by Mosier et al. (2012). The authors designed a fluid cell consisting of microfluidic channels that permitted to work on laminar flow, and utilized finite element analysis to profile fluid conditions during biofilm formation. In these conditions, the elastic modulus of $P$. aeruginosa PAO1 biofilms were obtained, ranging from 0.58 to $2.61 \mathrm{kPa}$ (Mosier et al., 2012). The technique also allowed measuring the mechanical properties of living, moving, and self-immobilized bacteria in physiological liquid medium.

Longo et al. (2012) showed with the so-called force volume methodology that the bacterial membrane of $E$. coli was not mechanically homogenous presenting areas of different compliance. In addition, they proposed the existence of stiffer structures below the membrane that could be associated with bacterial nucleoids.

Dhahri et al. did not use any immobilization protocol while studying the native gliding movements of Nostoc cyanobacteria (at $900 \mu \mathrm{m} / \mathrm{h}$ ). The authors used a procedure based on fast force-distance curves made at every pixel (reducing the lateral forces). They also study nonmotile Rhodococcus wratislaviensis. The obtained Young modulus and turgor pressure ranged, for both strains, from 20 to $105 \mathrm{MPa}$ and 40 to $310 \mathrm{kPa}$, respectively. Such investigations showed the possibility of studying phenomena such as bacterial propulsion and biofilm formation in real time at physiological conditions (Dhahri et al., 2013).

Another interesting creep experiment was performed this time to test the kinetics and mechanism of action of antimicrobial peptides on bacteria ( $\mathrm{Lu}$ et al., 2014). In this work, AFM helped to evaluate changes in the time-dependent mechanical properties of $P$. aeruginosa PAO1 cells after their treatment with antimicrobial peptides. Measurements carried out at different peptide concentrations revealed large changes in the viscoelastic parameters.

\section{Eukaryotic Cells}

By 2000, Rotsch and Radmacher were able to show the capability of AFM to monitor and quantify the effect of drugs on the elasticity of different components of the cytoskeleton of two fibroblast cell lines. The study pointed out the importance of the actin filaments for the mechanical stability of living cells, which were disassembled with Cytochalasins B and D and Latrunculin A. Interestingly, they reported that the disruption of microtubules did not seem to affect cell elasticity. The authors also used fluorescence microscopy to perform the experiments (Rotsch and Radmacher, 2000).

Already in 2003, the connection between AFM mechanical measurements and the possibility of detection, diagnosis, and treatment of disease was pointed out (Costa, 2003). AFM was also used to study the influence of the plasma-membrane receptor clustering 

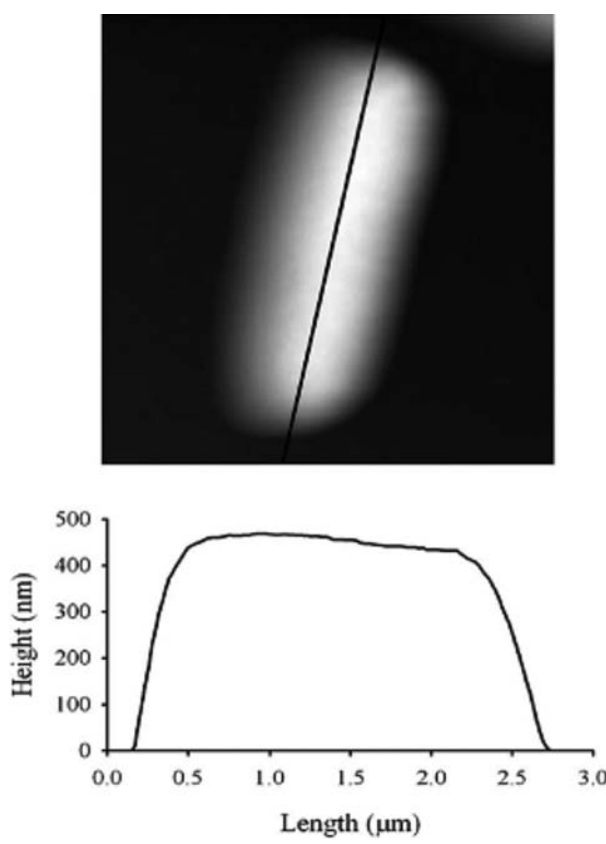

Fig. 3. Left: Deflection image $\left(2.5 \times 2.5 \mu \mathrm{m}^{2}\right)$ of a $P$. aeruginosa PAO1 cell taken at $1 \mathrm{nN}$. Right: Creep deformation as a function of time for pyramid-shaped tips (PT) and colloidal tips (CT) after apply-

on the local cell mechanics. The authors recorded maps of interaction forces between functionalized AFM-tips with antibodies and the vascular endothelial growth factor receptor VEGF, which concentrated toward the cell boundaries. They found an increase of fluidity under the clusters and a reduction in stiffness, which was proportional to the receptor density, providing an explanation for cell growth and angiogenesis (Almqvist et al., 2004).

The mechanics of cochlear outer hair cells (OHCs), which play a crucial role in the hearing organ, have also been investigated with AFM. The indentation measurements were consistent with the idea of shellcore ultrastructure, which seems to be important for the mechanics and electromotility of such cells. The cells did not present hysteresis at deformation rates of more than $40 \mu \mathrm{m} / \mathrm{s}$ suggesting that the $\mathrm{OHC}$ lateral wall is highly elastic, which could partly explain the rapid changes in shape that $\mathrm{OHCs}$ are believed to undergo in vivo (Zelenskaya et al., 2005).

The concept of "pointwise modulus" was introduced to improve the unrealistic assumptions of the Hertz theory (the model is discussed in the next section). With this approach, the authors examined human aortic endothelial cells (HAECs). The results showed that indentations in a range from 2 to $5 \mu \mathrm{m}^{2}$ in the cytoplasm revealed two different mechanical behaviors (for distinct cellular material) of values 5.6 and $1.5 \mathrm{kPa}$ at an indentation depth of $200 \mathrm{~nm}$. Treatment of the cells with Cytochalasin B led to a homogeneous linear elastic behavior (of value $0.89 \mathrm{kPa}$ ). This work pointed out that standard Hertz analysis was not the best model to explain the complex mechanical behavior of cells (Costa et al., 2006).

In 2007, Titushkin and Cho studied the mechanical properties of human mesenchymal cells (hMSCs) and

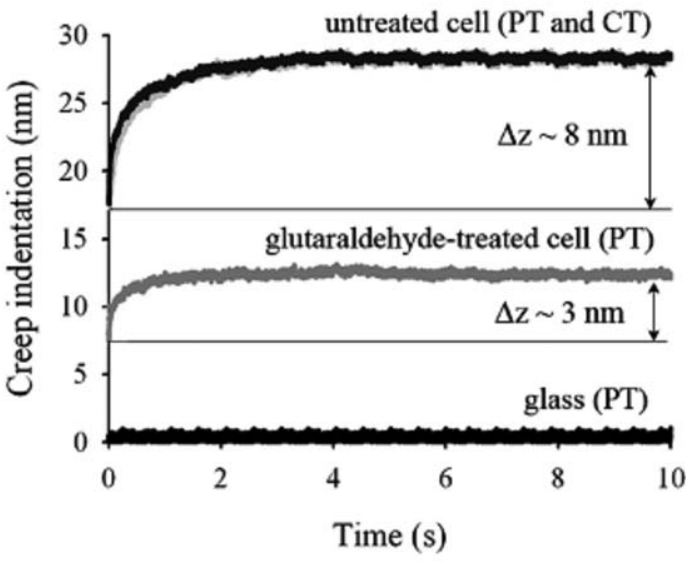

ing a loading force of $4 \mathrm{nN}$. The flat dark line (glass) does not creep with time (it does not deform). (Reproduced with permission of ASM from Vadillo-Rodriguez et al., J Bacteriol, 2008, 190, 4225-4232).

differentiated osteoblast, obtaining an average Young's modulus of 3.2 and $1.7 \mathrm{kPa}$, respectively. The authors concluded that the differences in cell elasticity and membrane mechanics resulted from a different actin cytoskeleton organization in these two cell types. It was also found that microtubules did not affect cellular mechanics. Furthermore, the authors postulated that the actin cytoskeleton had a pivotal role in the hMSC mechanical properties at the early stage of stem-cell differentiation (Titushkin and Cho, 2007).

Cross et al. (2007) investigated the mechanical properties of cells taken from patients with lung, breast, and pancreas cancer. This work is particularly interesting from the point of view of diagnostics, since the authors showed that for cells with similar shapes the mechanical analysis could discriminate normal from cancerous cells (in agreement with immunohistochemical testing).

Alveolar mechanics has also been studied with AFM and live cell fluorescence imaging (Azeloglu et al., 2008). The authors investigated three major cell types from the neonatal rat lung using the apparent depthdependent point wise elastic modulus (aforementioned). The results were in agreement with the reported heterogeneity of alveolar cell deformation during in situ lung inflation.

To gain understanding about the mechanisms that regulate the mechanical behavior of the cytoskeleton, Sunyer et al. (2009) performed mechanical essays with the AFM at different temperatures. The researchers measured the complex shear modulus of human alveolar epithelial cells over the frequency range (0.1-25.6 $\mathrm{Hz}$ ) varying the temperature from 13 to $37^{\circ} \mathrm{C}$. The findings indicated that the cells were stiffer (more solid-like) when the temperature was increased, concluding that the dependence of cell mechanics with 

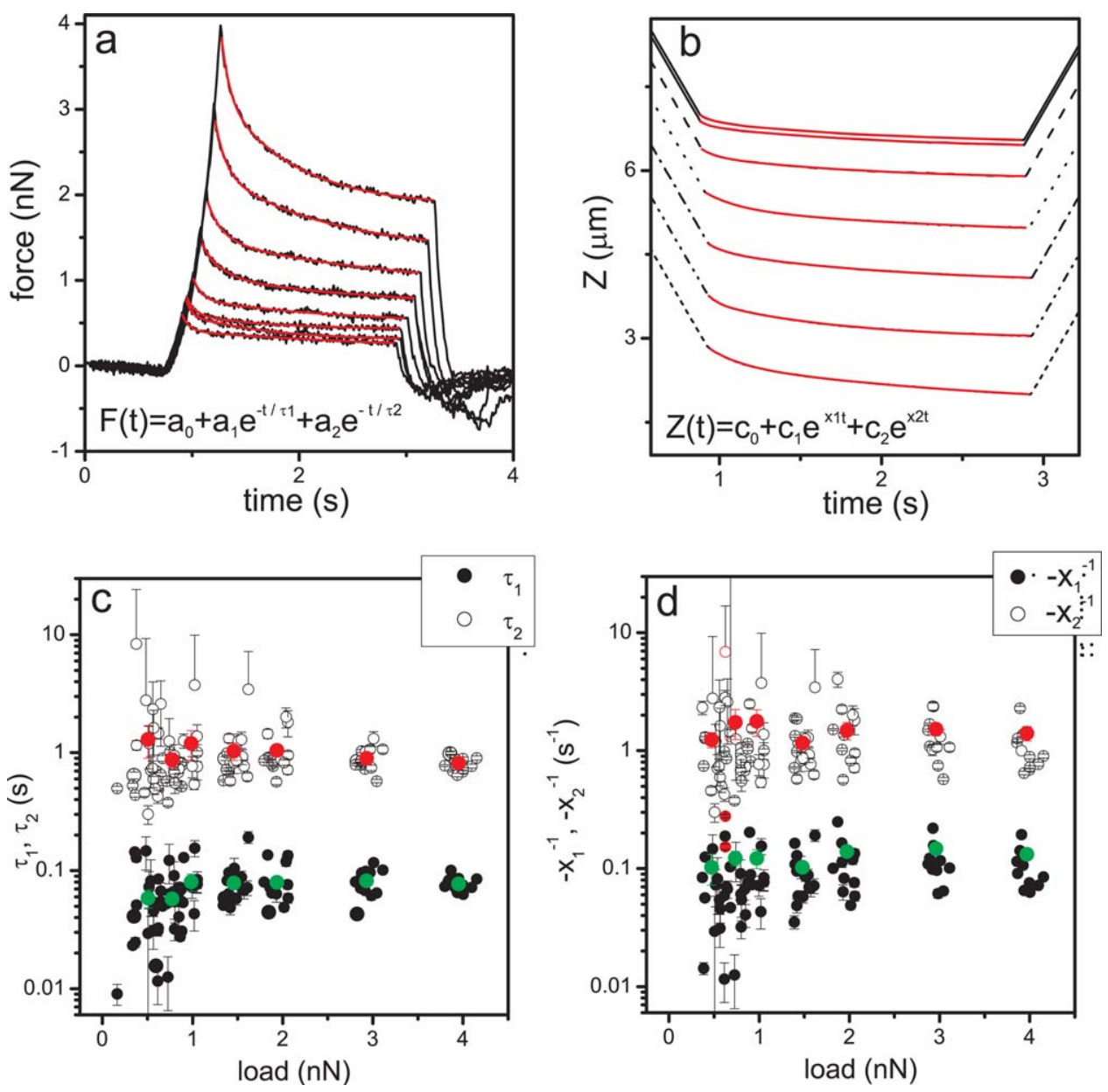

Fig. 4. Typical force relaxation (a) and creep (b) curves obtained at different loads taken on the nuclear region of an MCF-7 cell. Black lines correspond to experimental data while red lines show the fitting of the experimental data with the Zener's model (Riande et al., 2000). (c, d) Represent the relaxation and creep characteristic times, respec-

temperature might be dominated by the contractile activity of molecular motors (Sunyer et al., 2009).

In 2010, Moreno-Flores et al. presented a methodology based on stress relaxation to give account of the general complexity of the mechanical behavior of MCF-7 breast cancer cells. The methodology provided the cell relaxation response at nanometer level and permitted the elaboration of stress relaxation maps. The MCF-7 cell behavior could be well described by a generalized viscoelastic model. Within the range of applied forces $(0.5-4 \mathrm{nN})$ a slow and a fast relaxation with characteristic times of 0.1 and $1 \mathrm{~s}$ were observed, possibly due to rearrangements of cell membrane and cytoskeleton, respectively (Moreno-Flores et al., 2010a). The methodology was successfully implemented by including creep experiments (see Fig. 4), which showed that the mechanical behavior of MCF-7 cells responded to a two-layered model of similar elasticity but differing viscosity. The exposition of the cells to an actin-depolymerizing agent decreased the elasticity and the viscosity of the cell, being the cytoplasm the part that experienced the larger decrease (MorenoFlores et al., 2010b).

tively. The red and green points show their respective averages. (Reproduced with permission of IOP from Moreno-Flores et al., Nanotechnology, 2010b, 1, 445101). [Color figure can be viewed in the online issue, which is available at wileyonlinelibrary.com.]

In 2011, the role of keratin in cell mechanics was studied by Walter et al. The authors investigated the elastic behavior of pancreatic Panc-1 cells. The findings pointed out that the elastic modulus of the intact and skeletonized keratin network was about $10 \mathrm{~Pa}$, while the living cell elastic modulus varied from 100 to $500 \mathrm{~Pa}$. Furthermore, the authors stated that the large amount of keratin in these cells did not play a strong role on the elastic modulus of the cell actin might do (Walter et al., 2011).

How is the elasticity measurement influenced by the tip geometry? This question was addressed by Harris and Charras (2011). The authors found that pyramidal tips delivered values about twice larger than spherical tips (attributed to the larger contact area of the latter. The authors combined AFM with confocal microscopy to measure the indentation and characterize its geometry. The experiments were carried out on epithelial cells (which expressed a GFP-tagged membrane marker). The results showed that for pyramidal tips the contact area can be underestimated at forces larger than $0.2 \mathrm{nN}$. This could lead to an overestimation of elasticity and therefore it should be taken into account 

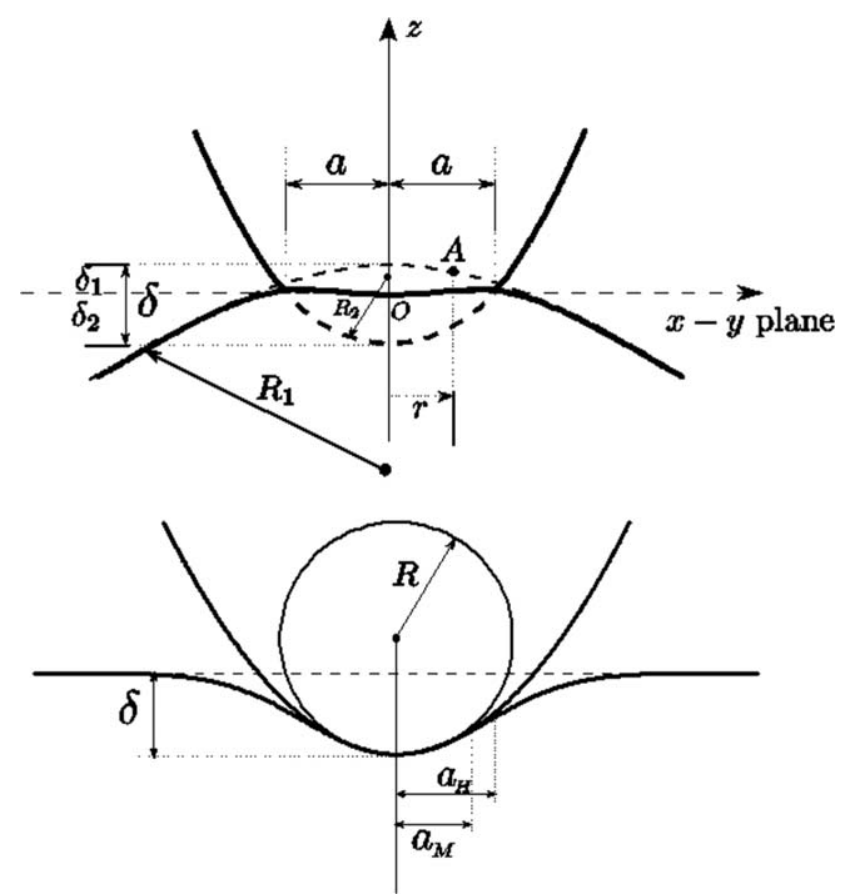

Fig. 5. Above: Schematic representation of two nonconforming surfaces approximated by two paraboloids of revolution. (Reproduced with permission from Johnson, Contact Mechanics, 1985, Cambridge University Press.) Below: Indentation of a rigid sphere tip into an elastic half space. For a large indentation, the differences in the contact radius between the Hertz $\left(\mathrm{a}_{\mathrm{H}}\right)$ parabolic approximation and the exact Maugis correction $\left(\mathrm{a}_{\mathrm{M}}\right)$ are no longer negligible.

in such type of experiments (Harris and Charras, 2011).

The impact of both actin and microtubulin filaments on the deformation of mouse ovarian cancer cells was studied by Ketene et al. Since nontumorigenic cells showed well-organized structures consisting of actin and microtubule filaments, while cells in aggressive cancer stages had disorganized actin and microtubule structures, the authors used actin targeting molecules in combination with anti-cancer drugs to modify the cell architectural framework. The results confirmed that the mechanical properties were clearly influenced by the organization state of the actin microfilaments (a decrease in the actin organization led to a decrease in cell elasticity and viscosity). On the contrary, the results showed that the influence of the microtubule organization on the mechanical properties of the cells was marginal (Ketene et al., 2012).

Interesting is the work by Stewart et al., which described an experimental protocol that can be generalized to globular cells in low-adhesive environments. Furthermore, the authors measured (with flat cantilevers and light microscopy) the mechanical stress and the volume under compression of HeLa mitotic cells (Stewart et al., 2012).

The changes in cortical and plasma mechanics during the process of cell spreading have been investigated with AFM and fluorescence microscopy (Pietuch and Janshoff, 2013). The authors found that over $2.5 \mathrm{~h}$ the cortical and membrane tension became constant at the expense of excess membrane area. They concluded that cell spreading was initiated by a drop in tension compensated by a decrease in excess area. Furthermore, they showed how to perturb the spreading process by adding molecular inhibitors.

The mechanical properties of bioartificial lungs reconstructed from decellularized organ scaffolds obtained with AFM seem to be of importance in the field lung transplantation. Luque et al. determined the local mechanical properties of the alveolar wall segments, alveolar wall junctions, and pleural regions. The authors found that the storage modulus of alveolar was about $6 \mathrm{kPa}$ while pleural regions were three-fold stiffer. This work suggests that local differences in mechanical properties could be relevant for the differentiation and function of lung cells (Luque et al., 2013).

An improvement in the understanding of the behavior of the cytoplasm, which is the largest part of the cell, has been reported recently (Moeendarbary et al., 2013). The results of the study validated the poroelastic model, which describes the cytoplasm as a biphasic material consisting of a porous elastic solid meshwork immersed in an interstitial fluid. The authors were able to show that water redistribution through the solid phase (e.g., cytoskeleton) had a crucial role in cellular rheology at short timescales.

A promising experimental alternative for future cell mechanics studies is the combination of AFM with fluorescence superresolution techniques such as stimulated emission depletion microscopy (STED) and sstochastic optical reconstruction microscopy. The main advantage is that fluorescence superresolution techniques might localize selected molecular species with a resolution below the diffraction limit, complementing in this way, the morphological information provided by AFM. On this context, Varghese Chacko et al (2013) showed that this combination was able to deliver accurate topological features and mechanical information (Young modulus) at the nanoscale of fibroblast cells (Varghese Chacko et al., 2013).

This section will close with the mention of an interesting experiment that connects cell compression with cytotoxicity (Zimmer et al. 2014). In this work, the authors found that $\mathrm{ZnO}$ nanoparticles caused significant changes in the force-deformation profiles while other nanoparticles like $\mathrm{SiO}_{2}$ did not produce such effect. This type of experiments opens a way to study the effect of (toxic) nanoparticle on the physical and biological properties of cells.

\section{MECHANICAL MODELS: THE MICRO AND NANO CONNECTION}

In this section, we introduce and discuss two mechanical models commonly used to explain the results obtain with AFM. Maybe the most used model in soft matter mechanics is derived from the Hertz theory of elastic contact (Johnson, 1985). The classical Hertz theory describes the contact of two nonconforming elastic surfaces (see Fig. 5). The main assumptions of the Hertz theory can be summarized as follows: (i) The two surfaces are nonconforming and the region of contact has an area whose characteristic size $a$, is much smaller than the radius of curvature of the surfaces, (ii) as a consequence of (i), the strains are small and each surface can be considered as a half space, and (iii) The contact is frictionless. 
The theory provides the deformed surface profiles as well as the pressure distributions, which is equivalent to knowing the stress and strain distributions for a given load. For solids of revolution, which play a fundamental role in AFM experiments, the pressure distribution obtained is given by:

$$
p(r)=p_{0} \sqrt{1-\frac{r^{2}}{a^{2}}}
$$

From this equation, the main features of the contact problem can be derived, that is, the radius of contact, $a$, and the maximum contact pressure, $p_{0}$

$$
a=\left(\frac{3 P R}{4 E^{*}}\right)^{1 / 3} ; \quad p_{0}=\frac{3 P}{2 \pi a^{2}}=\left(\frac{6 P E^{* 2}}{\pi^{3} R^{2}}\right)^{1 / 3} .
$$

Being $P$, the total load compressing the surfaces, $R$, the relative curvature radius, related to the surfaces curvatures radii by $(1 / R)=\left(1 / R_{1}+1 / R_{2}\right)$, and $E^{*}=$ $\left(\frac{1-v_{1}^{2}}{E_{1}}+\frac{1-v_{2}^{2}}{E_{2}}\right)^{-1}$ a relative Young modulus.

A second theory, also used to interpret mechanical properties of soft materials is the Johnson-KendallRoberts theory of adhesive elastic contact (Johnson, 1985). The Hertz theory does not consider adhesive forces, that is, the force falls to zero when the load is removed and the surfaces are no longer in contact. Nevertheless, experimental evidences indicate the contrary: when the load is removed, and the two surfaces separate from contact, negative forces due to adhesion appear. To take into account adhesive interactions in the contact regime, the JKR theory, making the same basic assumptions as the Hertz theory, splits the total free energy into the sum of two components: the stored elastic strain energy and the surface energy due to adhesive forces:

$$
U_{T}=U_{E}+U_{S}
$$

Keeping the total compression, $\delta$, constant, at equilibrium we have that $\left[\frac{\partial U_{T}}{\partial a}\right]_{\delta=\text { const }}=0$, and thus,

$$
\left[\frac{\partial U_{E}}{\partial a}\right]_{\delta}=-\left[\frac{\partial U_{S}}{\partial a}\right]_{\delta} .
$$

The elastic energy can be obtained from the general solution for the pressure distribution:

$$
p(r)=p_{0} \sqrt{1-\frac{r^{2}}{a^{2}}}+p_{0}^{\prime} \frac{1}{\sqrt{1-\frac{r^{2}}{a^{2}}}} .
$$

In the Hertz theory, the coefficient $p_{0}^{\prime}$ was assumed to vanish, since in the case it is positive, the second term of the RHS of this pressure distribution produces infinite gradients at the radius of contact. Thus, only negative values of $p_{0}^{\prime}$ could lead to finite pressure gradients. Such negative values for the pressure are due to adhesion forces. In the Hertz theory, these adhesion phenomena were not considered and therefore the second term was neglected.

Computing the total work due to this pressure distribution, we obtain the elastic energy

$$
U_{E}=\frac{\pi^{2} a^{3}}{E^{*}}\left(\frac{2}{15} p_{0}^{2}+\frac{2}{3} p_{0} p_{0}^{\prime}+p_{0}^{2}\right)
$$

The total compression can be calculated then, using the same equations as in the Hertz theory, with the new pressure distribution, obtaining

$$
\delta=\frac{\pi a}{2 E^{*}}\left(p_{0}+2 p_{0}^{\prime}\right)
$$

However, the adhesion surface energy is considered to be proportional to the contact area,

$$
U_{S}=-2 \gamma \pi a^{2} \text {. }
$$

Being $\gamma$, the Dupré energy of adhesion (as a surface energy per unit area of each surface). At equilibrium, we have that, $\left[\frac{\partial U_{E}}{\partial a}\right]_{\delta}=-\left[\frac{\partial U_{S}}{\partial a}\right]_{\delta}$, therefore substituting the corresponding quantities, and chosen the sign so there are no compressive stresses at $r=a$, we find that the adhesive pressure is given by $p_{0}^{\prime}=-\sqrt{\frac{4 \gamma E^{*}}{\pi a}}$.

The relationship between the net contact load $P$ and the contact radius $a$ is then

$$
\left(P-\frac{4 E^{*} a^{3}}{3 R}\right)^{2}=16 \pi \gamma E^{*} a^{3},
$$

which can be rewritten as

$$
a^{3}=\frac{3 R}{4 E^{*}}\left(P+3 \pi \gamma R+\sqrt{6 \pi \gamma R P+(3 \pi \gamma R)^{2}}\right) .
$$

That is, the contact radius is given by the one predicted by the Hertz theory with an adhesive correction given by $\frac{3 R}{4 E^{*}}\left(3 \pi \gamma R+\sqrt{6 \pi \gamma R P+(3 \pi \gamma R)^{2}}\right)$.

\section{The Maugis Correction for Large Deformations}

The two theories described above assume the hypothesis that the contact radius is much smaller than the size of the particles involved. This is evidenced by the approximation of the shape of the surface by a paraboloid of revolution. For the case of the contact of two nonconforming spheres, JKR and Hertz theories used the parabolic approximation for the shape of a sphere of radius $R, f(r)=r^{2} / 2 R$. If the indentation (i.e., the contact radius $a$ ) is not small compared with $R$, then such parabolic approximation is no longer valid. Maugis (1995) used the exact expression for the shape of the sphere $f(r)=R-\sqrt{R^{2}-r^{2}}$ (see Fig. $5)$. With such modification, the equilibrium relations $\delta(a)$, and $P(a)$, are given by

$$
\begin{gathered}
\delta(a)=\frac{a}{2} \ln \frac{R+a}{R-a}-\sqrt{\frac{2 \gamma \pi a}{E^{*}}} \\
P(a)=2 a E^{*}\left(\frac{R^{2}+a^{2}}{4 a} \ln \frac{R+a}{R-a}-\frac{R}{2}-\sqrt{\frac{2 \gamma \pi a}{E^{*}}}\right)
\end{gathered}
$$

These relations show that the JKR approximation is valid only for large values of the radius [for values of $\left.\left(R E^{*}\right) /(\pi \gamma)>750\right]$. 


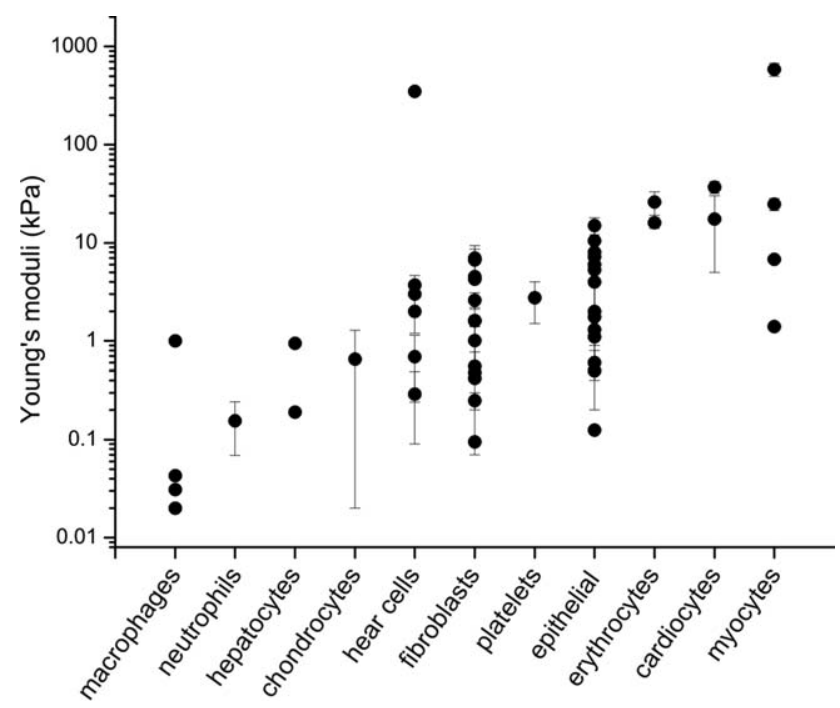

Fig. 6. The figure shows the values of the modulus of Young for different cells, which are often treated as being elastic. (Reproduced with permission from Melzak et al., Soft Matter, 2011, 7, 332-342, Royal Society of Chemistry.)

\section{Dufresne Approach to Microscale Indentations}

The JKR theory together with the Maugis correction, describe very accurately the elastic adhesive contact of two soft surfaces; however, it is developed as a macroscopic mean field theory, and therefore, the use of this theory in microscopic systems may lead to divergences between the predictions and the experimental data. Recently, Style et al. reported that for small contact radius, solid surface tension should be taken into account since a considerable deviation from both the JKR and Maugis theories was found (Style et al., 2013). For a contact between a rigid sphere $\left(E_{1}=\infty\right)$ and radius between 3 and $30 \mu \mathrm{m}$ and a soft substrate, it was found that JKR theory worked only for the stiffer substrates (down to $E=85 \mathrm{kPa}$ ), but it failed to predict both the contact radius and the indentation in the case of very soft substrates $(E=3 \mathrm{kPa})$. Even with the Maugis correction for large deformation, a strong systematic deviation from observation was found. To explain these discrepancies between the predicted and the observed data, an energy balance argument was used. The total energy change as a result of the indentation of the rigid sphere into the soft substrate is split into three parts: the elastic energy, $U_{e l}$, described by the Hertz theory, the surface tension energy, $U_{\gamma}$, as a result of the stretching of the substrate surface, which increases its area, and the adhesion energy, $U_{a d}$. Approximating the indentation as a spherical cap into a flat plane, the total energy is then given by

$$
U=U_{e l}+U_{a d}+U_{\gamma}=c E^{*} \sqrt{R \delta^{5}}+\pi \gamma_{s v} \delta^{2}-2 \pi \gamma R \delta,
$$

where $c$ is a proportionality constant and $\gamma_{s v}$ is the work needed to create new surface area in the substrate by stretching. At equilibrium, we have a balance equation

$$
\frac{5}{2} c E^{*} \sqrt{R \delta^{3}}+2 \pi \gamma_{s v} \delta=2 \pi \gamma R .
$$

If $R$ is very large, in the LHS of the balance equation, the surface tension term is negligible compared with the elastic term and, thus, for $c=8 /(5 \sqrt{3})$ the JKR theory result is recovered. However, if $R$ is small, then the elastic term is the one which can be neglected and then the tension surface term balances the adhesion energy, obtaining the indentation

$$
\delta=\frac{\gamma R}{\gamma_{s v}} .
$$

\section{CONCLUSIONS AND OUTLOOK}

This review has introduced the use of the AFM in the complex world of cell mechanics. Part of the aim of the review was to show the potential that AFM has to investigate basic questions of biology or soft matter physics, and how the researcher can obtain a phenomenological answer.

The technique, up to date, has been able to deliver not only high resolution cell images but also quantitative information about the cell surface properties, the Young modulus, the viscosity or the relaxation times. However, as Figure 6 shows (Melzak et al., 2011), the scatter of the Young modulus for similar cells indicates that the topic is complex and more experimental investigations and theoretical modeling are needed.

How cells behave as a whole? Which is the response of a cell constituent to an external chemical or mechanical input? Whatever the scientific question might be, the AFM by itself will not be able to provide this information, and therefore its combination with techniques that can deliver chemical fingerprints simultaneously with the cell physical properties would be desirable. This short review has not addressed the use of AFM on plant cells; a field that is developing quickly due to its biotechnological applications and that deserves a manuscript on its own.

At this point, the reader should be aware that there is plenty of room at the bottom and at the top, room for experimentalist and theoreticians that aim to understand the behavior of cells and its components at the microscale and nanoscale.

*In certain AFMs, the tip is fixed and the piezoscanner moves the sample in the three dimensions (e.g., the old Multimode from Veeco, now Bruker).

\section{ACKNOWLEDGMENTS}

JLTH would like to thank his group for the work done on soft matter mechanics since 2008. The authors thank M. dM Vivanco (BioGUNE, Derio, Spain) for providing MCF-7 cells.

\section{REFERENCES}

Alcaraz J, Buscemi L, Grabulosa M, Trepat X, Fabry B, Farre R Navajas D. 2003. Microrheology of human lung epithelial cells measured by atomic force microscopy. Biophysical J 84:2071-2079.

Alessandrini A, Facci P. 2005. AFM: A versatile tool in biophysics. Meas Sci Technol 16:R65-R92.

Almqvist N, Bhatia R, Primbs G, Desai N, Banerjee S, Lal R. 2004. Elasticity and adhesion force mapping reveals real-time clustering of growth factor receptors and associated changes in local cellular rheological properties. Biophys J 86:1753-1762.

Arnoldi M, Fritz M, Bauerlein E, Radmacher M, Sackmann E, Boulbitch A. 2000. Bacterial turgor pressure can be measured by atomic force microscopy. Phys Rev E 62:1034-1044.

Azeloglu EU, Bhattacharya J, Costa KD. 2008. Atomic force microscope elastography reveals phenotypic differences in alveolar cell stiffness. J Appl Physiol 105:652-661. 
Benitez R, Moreno-Flores S, Bolos V, Toca-Herrera JL. 2013. A New automatic contact point detection algorithm for AFM force curves. Microsc Res Tech 76:870-876.

Borkovec M, Szilagyi I, Popa I, Finessi M, Sinha P, Maroni P, Papastavrou G. 2012. Investigating forces between charged particles in the presence of oppositely charged polyelectrolytes with the multi-particle colloidal probe technique. Adv Colloid Interface Sci 179-182:85-98

Bornschlögl T, Rief M. 2011. Single-molecule protein unfolding and refolding using atomic force microscopy. Methods Mol Biol 783 233-250.

Braunstein D, Spudicht A, 1994. Structure and activation dynamics of RBL-2H3 cells observed with scanning force microscopy. Biophys $\mathrm{J}$ 66:1717-1725.

Camesano TA, Natan MJ, Logan B E. 2000. Observation of changes in bacterial cell morphology using tapping mode atomic force microscopy. Langmuir 16:4563-4572.

Costa KD. 2003. Single-cell elastography: Probing for disease with the atomic force microscope. Dis Markers 19:139-154

Costa KD, Sim AJ, Yin FC. 2006. Non-Hertzian approach to analyzing mechanical properties of endothelial cells probed by atomic force microscopy. J Biomech Eng 128:176-184.

Crick SL, Yin FC. 2007. Assessing micromechanical properties of cells with atomic force microscopy: Importance of the contact point. Biomech Model Mechanobiol 6:199-210.

Cross SE, Jin, YS, Rao J, Gimzewski JK. 2007. Nanomechanical analysis of cells from cancer patients. Nat Nanotechnol 2:780-783.

Cumpsen PJ, Clifford CA, Portoles JF, Johnstone JE, Munz M. 2008. Cantilever spring-constant calibration in atomic force microscopy. In: Bhushan B, Fuchs H, Tomitori M, editors. Applied scanning probe methods, Vol. 8. Heidelberg: Springer.

Doktycz MJ, Sullivan CJ, Hoyt PR, Pelletier DA, Wu S, Allison DP. 2003. AFM imaging of bacteria in liquid media immobilized on gelatin coated mica surfaces. Ultramicroscopy 97:209-216.

Dhahri S, Ramonda M, Marliere C. 2013. In-situ determination of the mechanical properties of gliding or non-motile bacteria by atomic force microscopy under physiological conditions without immobilization. PLoS One 8:e61663.

Ducker WA, Senden TJ, Pashley RM. 1991. Direct measurement of colloidal forces using an atomic force microscope. Nature 353:239241.

Fischel A. 1906. The developmental history of echinoderms. I. The mechanics of cells division. II. Experiments with vital coloration. Archiv für Entwiclungsmechanick der Organismen 22:526-554

Fisher TE, Oberhauser AF, Carrion-Vazquez M, Marszalek PE, Fernandez JM. 1999. The study of protein mechanics with the atomic force microscope. Trends Biochem Sci 24:379-384.

Florin E-L, Moy VT, Gaub HE. 1994. Adhesive forces between individual ligand receptor pairs. Science 264:415-417.

Gaboriaud F, Bailet S, Dague E, Jorand F. 2005. Surface structure and nanomechanical properties of Shewanella putrefaciens bacteria at two $\mathrm{pH}$ values $(4$ and 10 ) determined by atomic force microscopy. J Bacteriol 187:3864-3868.

Garcia R, Tamayo J, San Paulo A. 1999. Phase contrast and surface energy hysteresis in tapping mode scanning force microscopy. Surf Interface Anal 27:312-316.

Harris AR, Charras GT. 2011. Experimental validation of atomic force microscopy-based cell elasticity measurements. Nanotechnology $22: 345102$.

Henderson E, Haydon PG, Sakaguchi DS. 1992. Actin filament dynamics in living glial cells imaged by atomic force microscopy. Science 257:1944-1946.

Hinterdorfer P, Dufrêne YF, 2006. Detection and localization of single molecular recognition events using atomic force microscopy. Nat Methods 3:347-355.

Hobbs JK, Farrance OE, Kailas L, 2009. How atomic force microscopy has contributed to our understanding of polymer crystallization. Polymer 50:4281-4292.

Hoh JH, Schoenenberger C-A. 1994. Surface morphology and mechanical properties of MDCK monolayers by atomic force microscopy. J Cell Sci 107:1105-1114.

Hugel T, Seitz M. 2001. The study of molecular interactions by AFM force spectroscopy. Macromol Rapid Commun 22:989-1016.

Johnson KL. 1985. Contact Mechanics. Cambridge: Cambridge University Press.

Ketene AN, Roberts PC, Shea AA, Schmelz EM, Agah M. 2012. Actin filaments play a primary role for structural integrity and viscoelastic response in cells. Integr Biol 4:540-549.

Le Grimellec Ch, Lesniewska, E, Giocondi M-C, Finot E, Vie V, Goudonnet J-P. 1998. Imaging of the surface of living cells by lowforce contact-mode atomic force microscopy. Biophys J 75:695-703.
Longo G, Rio LM, Roduit C, Trampuz A, Bizzini A, Dietler G, Kasas S. 2012. Force volume and stiffness tomography investigation on the dynamics of stiff material under bacterial membranes. J Mol Recognit 25:278-284

Lu S, Walters G, Parg R, Dutcher JR. 2014. Nanomechanical response of bacterial cells to cationic antimicrobial peptides. Soft Matter 10:1806-1815.

Luque T, Melo E, Garreta E, Cortiella J, Nichols J, Farre R, Navajas D. 2013. Acta Biomater 9:6852-6859.

Maugis D. 1995. Extension of the Johnson-Kendall-Roberts theory of the elastic contact of spheres to large contact radii. Langmuir 11: 679-682.

Melzak KA, Moreno-Flores S, Yu K, Kizhakkedathu J, TocaHerrera JL. 2010. Rationalized approach to the determination of contact point in force-distance curves: Application to polymer brushes in salt solutions and in water. Microsc Res Tech 73:959964.

Melzak KA, Moreno-Flores S, Eleta Lopez A, Toca-Herrera JL. 2011. Why size and speed matter: Frequency dependence and the mechanical properties of biomolecules. Soft Matter 7:332-342.

Melzak KA, Lazaro GR, Hernandez-Machado A, Pagonabarraga I, Cardenas Diaz de Espada JM, Toca-Herrera JL. 2012. AFM measurements and lipid rearrangements: Evidence from red blood cell shape changes. Soft Matter 8:3716-3726.

Mendez-Vilas A, Gallardo-Moreno AM, Gonzalez-Martin ML. 2006. Nano-mechanical exploration of the surface and sub-surface of hydrated cells of Staphylococcus epidermidis. Anton Leeuw 89 373-386.

Moeendarbary E, Valon L, Fritzsche M, Harris A R, Moulding DA Thrasher AJ, Stride E, Mahadevan L, Charras GT. 2013. The cytoplasm of living cells behaves as a poroelastic material. Nat Mater $12: 253-261$.

Moreno-Flores S, Benitez R, Vivanco MdM, Toca-Herrera JL. 2010a. Stress relaxation microscopy: Imaging local stress in cells. J Biomechanics 43:349-354.

Moreno-Flores S, Benitez R, Vivanco MdM, Toca-Herrera JL, 2010b. Stress relaxation and creep on living cells with the atomic force microscope: A means to calculate elastic moduli and viscosities of cell components. Nanotechnology 1:445101.

Moreno Flores S, Toca-Herrera JL. 2013. Hybridizing Surface Probe Microscopes: Toward a Full Description of the Meso- and Nanoworlds. Boca Raton, FL: CRC Press.

Mosier AP, Kaloyeros AE, Cady NC. 2012. A novel microfluidic device for the in situ optical and mechanical analysis of bacterial biofilms. J Microbiol Methods 91:198-204.

Murphy WL, Mercurius KO, Koide S, Mrksich M. 2004. Substrates for cell adhesion prepared via active site-directed immobilization of a protein domain. Langmuir 20:1026-1030.

Pietuch A, Janshoff A. 2013. Mechanics of spreading cells probed by atomic force microscopy. Open Biol 3:130084.

Puech PH, Poole K, Knebel D, Muller DJ. 2006. A new technical approach to quantify cell-cell adhesion forces by AFM. Ultramicroscopy 106:637-644.

Putman CAJ, van der Werf KO, de Grooth BG, van Hulst NF, Greve J. 1994. Viscoelasticity of living cells allows high resolution imaging by tapping mode atomic force microscopy. Biophys J 67:17491753

Radmacher M, Tillamnn RW, Fritz M, Gaub HE. 1992. From molecules to cells: Imaging soft samples with the atomic force microscope. Science 257:1900-1905.

Radmacher M, Fritz M, Kacher CM, Cleveland JP, Hansma PK. 1996. Measuring the viscoelastic properties of human platelets with the atomic force microscope. Biophys J 70:556-567.

Radmacher M. 2002. Measuring the elastic properties of living cells by the atomic force microscope. Methods Cell Biol 68:67-90.

Raman A, Trigueros S, Cartagena A, Stevenson AP, Susilo M, Nauman E, Contera SA. 2011. Mapping nanomechanical properties of live cells using multi-harmonic atomic force microscopy. Nat Nanotechnol 6:809-814.

Riande E, Diaz-Calleja R, Prolongo MG, Masegosa RM, Salom C. 2000. Polymer Viscoelasticity. Stress and Strain in Practice. New York: Dekker. pp. 879.

Rotsch C, Radmacher M. 2000. Drug-induced changes of cytoskeletal structure and mechanics in fibroblasts: An atomic force microscopy study. Biophys J 78:520-535.

Saravia V, Toca-Herrera JL. 2009. Substrate Influence on Cell Shape and Cell Mechanics: HepG2 Cells Spread on Positively Charged Surfaces. Microsc Res Tech 72:957-964.

Scheuring S, Dufrene YF. 2010. Atomic force microscopy: Probing the spatial organization, interactions and elasticity of microbial cell envelopes at molecular resolution. Mol Microbiol 75:1327-1336. 
Stewart M, Toyoda Y, Hyman AA, Müller DJ. 2012. Tracking mechanics and volume of globular cells with atomic force microscopy using a constant-height clamp. Nature Protocols 7:143-154.

Style RW, Hyland C, Boltyanskiy R, Wettlaufer JS, Dufresne ER. 2013. Surface tension and contact with soft elastic solids. Nat Commun 4:2728.

Sunyer R, Trepat X, Fredberg JJ, Farre R, Navajas D. 2009. The temperature dependence of cell mechanics measured by atomic force microscopy. Phys Biol 6:025009.

Titushkin I, Cho M. 2007. Modulation of cellular mechanics during osteogenic differentiation of human mesenchymal stem cells. Biophys J 93:3693-3702.

Vadillo-Rodriguez V, Beveridge TJ, Dutcher JR. 2008. Surface viscoelasticity of individual gram-negative bacterial cells measured using atomic force microscopy. J Bacteriol 190:4225-4232.

Van Landingham MR, Villarrubia JS, Guthrie WF, Meyers GF. 2001. Nanoindentation of polymers: An overview. Macromol Symp 167 15-43.
Vargas-Pinto R, Gong H, Vahabikashi A, Johnson M. 2013. The effect of the endothelial cell cortex on atomic force microscopy measurements. Biophys J 105:300-309.

Varghese Chacko J, Cella Zanacchi F, Diaspro A. 2013. Probing cytoskeletal structures by coupling optical superresolution and AFM techniques for a correlative approach. Cytoskeleton 70:729740 .

Walter N, Busch T, Seufferlein T, Spatz JP. 2011. Elastic moduli of living epithelial pancreatic cancer cells and their skeletonized keratin intermediate filament network. Biointerphases 6:79-85.

Zelenskaya A, de Monvel JB, Pesen D, Radmacher M, Hoh JH, Ulfendahl M. 2005. Evidence for a highly elastic shell-core organization of cochlear outer hair cells by local membrane indentation. Biophys J 88:2982-2993.

Zimmer CC, Liu YX, Morgan JT, Yang G, Wang KH, Kennedy IM, Barakat AI, Liu GY. 2014. A New Approach to Investigate the Cytotoxicity of Nanomaterials Using Single Cell Mechanics. J Phys Chem 118:1246-1255. 